Relations industrielles

Industrial Relations

\title{
L’appréciation des politiques de D.G.B. par les syndicats français
}

How French Labour Unions View the Policies of the D.G.B.

\section{François Sellier}

Volume 33, numéro 3, 1978

URI : https://id.erudit.org/iderudit/028887ar

DOI : https://doi.org/10.7202/028887ar

Aller au sommaire du numéro

Éditeur(s)

Département des relations industrielles de l'Université Laval

ISSN

0034-379X (imprimé)

1703-8138 (numérique)

Découvrir la revue

Citer cet article

Sellier, F. (1978). L'appréciation des politiques de D.G.B. par les syndicats français. Relations industrielles / Industrial Relations, 33(3), 406-417.

https://doi.org/10.7202/028887ar

\section{Résumé de l'article}

Cet article a pour objet d'explorer et de faire comprendre les divergences entre les divers systèmes de relations professionnelles en Europe de l'Ouest, principalement entre les systèmes français et allemand.
Tous droits réservés (C) Département des relations industrielles de l'Université Laval, 1978
Ce document est protégé par la loi sur le droit d'auteur. L'utilisation des services d'Érudit (y compris la reproduction) est assujettie à sa politique d'utilisation que vous pouvez consulter en ligne.

https://apropos.erudit.org/fr/usagers/politique-dutilisation/ 


\section{L'appréciation des politiques du D.G.B. par les syndicats français}

\section{François Sellier}

Cet article a pour objet d'explorer et de faire comprendre les divergences entre les divers systèmes de relations professionnelles en Europe de l'Ouest, principalement entre les systèmes français et allemand.

Comparer les systèmes de relations industrielles de deux pays différents est toujours une tâche difficile car chacun des deux pays se caractérise par un environnement social global auquel son système est adapté, si bien que chacun des éléments de ce système ne peut s'expliquer que par les caractères spécifiques de la société toute entière. Lorsqu'un syndicaliste français - ou allemand - porte un jugement sur telle institution particulière de l'autre pays, (le comité d'entreprise pour la France et le Betriebsrat pour l'Allemagne, par exemple) il compare ce qui est incomparable dans la mesure où l'histoire et le fonctionnement de ces deux institutions ne peuvent se comprendre que comme la partie d'un vaste ensemble.

Et cependant, ces systèmes remplissent des fonctions qui ont quelque chose de commun: la défense des travailleurs, et pour les syndicats, d'une part, la tentative d'obtenir davantage de contrôle sur le pouvoir de décision des employeurs, et d'autre part, d'influencer le système législatif du travail et le système économique. De ce point de vue, on retrouve toujours trois centres de pouvoir: l'entreprise, l'État et les syndicats, le «pouvoir» de ces derniers étant toujours plutôt de l'ordre de l'influence sur la décision plutôt que pouvoir de décision proprement dit. Enfin, dans la mesure où les partis politiques luttent pour obtenir le pouvoir législatif, les syndicats ne peuvent être indifférents à leurs options et aux moyens de les influencer - ou de les utiliser - la réciproque pouvant exister.

D'un côté, par conséquent, il est bien vrai qu'on peut comparer l'efficacité des syndicats à obtenir des lois favorables à la sécurité d'emploi et à l'amélioration des salaires et des conditions de travail.

* SELliER, François. Centre national de la recherche scientifique, Paris. 
Mais, il est plus difficile de porter un jugement sur les différences de méthodes car les méthodes dépendent de l'environnement social. Toutefois, certaines circonstances peuvent rendre plus facile cette appréciation mutuelle: c'est le cas lorsqu'une crise économique affecte de la même façon l'économie et les travailleurs dans plusieurs pays, et c'est bien la situation, en Europe, aujourd'hui. Alors, les syndicats sont amenés par la ressemblance des contraintes qui s'exercent sur eux, par le rapprochement de leurs objectifs immédiats (et par l'importance croissante de ces objectifs immédiats relativement aux objectifs plus lointains), à s'observer plus attentivement et à mieux se comprendre parce que ce qui les distingue perd de l'importance par rapport à ce qui leur est commun.

Il semble qu'en France, actuellement, se développe une meilleure compréhension des syndicats allemands et se prépare un climat permettant une meilleure coopération: "l'unité d'action syndicale en Europe occidentale est devenue une nécessité sans laquelle le mouvement syndical n'est plus en mesure d'assumer les responsabilités qui lui incombent et de défendre les intérêts des travailleurs dont il a la charge», déclarait déjà en 1975 Georges Séguy, le secrétaire général de la CGT $^{1}$. Et, à la CFDT, le rapport du Congrès de la Fédération de la Métallurgie notait: «les divergences s'atténuent actuellement, du fait de la crise, commune à tous les pays... Certaines organisations syndicales de pays riches, qui traditionnellement ne contestaient pas en profondeur le système dans lequel elles vivaient, se sont elles aussi interrogées et ont engagé la lutte (les États Unis, l'Allemagne) $»^{2}$. Et la Confédération FO, dans le rapport à son 13ème Congrès, déclarait: «Ce système a prouvé son incapacité à respecter la dignité de l'homme et son droit imprescriptible à un travail rémunérateur... F.O. veut intensifier son action au sein de la Confédération Internationale des Syndicats libres pour l'élaboration d'une charte du développement que seule la solidarité ouvrière peut imposer aux États... Le congrès estime qu'une coopération étroite entre les pays de l'Europe pour définir un programme de développement social... apparaît comme un élément indispensable pour l'ouverture sur un monde moins injuste. FO travaillera dans ce sens au sein de la Confédération Européenne des syndicats ».

Bien que les divergences bien connues demeurent entre les syndicats, y compris du point de vue de la politique européenne, il est

Le Peuple, 1-31 Juillet 1975, p. 18.

2 FGM-CFDT, Bulletin du Militant, $\mathrm{n}^{\circ}$ 73, novembre 1977, p. 41. 
significatif de noter, partout, un net élargissement des préoccupations vers la coopération syndicale européenne. Cet élargissement se manifeste d'abord par des contacts plus nombreux. Ces contacts, et la perspective nouvelle décrite ci-dessus créent un nouveau climat de compréhension que nous examinons d'abord du point de vue de la pratique revendicative, ensuite du point de vue des attitudes à l'égard de la participation à la gestion.

\section{EFFORTS POUR UN ACCROISSEMENT DES COMMUNICATIONS ENTRE LES SYNDICATS FRANÇAIS ET ALLEMANDS}

On sait que la CGT a demandé déjà depuis plusieurs années son admission à la Confédération Européenne des Syndicats et que l'obstacle à son entrée semble venir de son adhésion à la Fédération Syndicale Mondiale. Cependant, la CGT poursuit ses efforts pour une telle admission. Pour se faire mieux reconnaître comme le plus puissant des syndicats français, elle vient d'éditer une brochure bien documentée, en allemand ${ }^{3}$, dont nous extrayons ce passage de la conclusion: «quant au Marché Commun, aux entreprises multinationales et aux monopoles, la CGT lutte pour l'établissement de l'Unité d'action des travailleurs en Europe et défend, dans les instances officielles de la Communauté Économique Européenne les revendications et les intérêts des travailleurs. La candidature à la Confédération Européenne des Syndicats correspond aux mêmes efforts ».

Les entreprises multinationales sont aussi, pour les syndicats, une occasion de rencontre ou de travail commun et la CGT, dans la métallurgie, y noue des contacts informels assez régulièrement. L'appartenance de la CGT à la Fédération Syndicale mondiale l'isole des Syndicats Professionnels Internationaux mais un des syndicats d'industrie de la CGT, la Fédération du Livre, adhère à la Fédération Internationale des Arts Graphiques qui rassemble tous les syndicats européens. La CGT est également active au Bureau International du Travail.

À la CFDT, les contacts entre syndicats d'industrie européens, spécialement du Métal, de la Chimie et de l'Alimentation, ont été fréquents depuis plusieurs années, et se sont accrus depuis deux ans. C'est le cadre de la CES et des Syndicats Professionnels Internationaux qui a été le plus propre à la multiplication de ces contacts. On note un souci particulier de mieux connaître le DGB. Des stages de rencontre entre jeunes syndicalistes ont été organisés par l'inter-

3 HERAUSGEBER: CGT, 213 rue Lafayette, 75480 Paris, Cedex 10. 
médiaire de l'Office Franco-allemand de la Jeunesse et, dans certains cas, la CFDT a pu désigner certains responsables syndicaux jeunes pour participer à ces échanges. Nous avons d'ailleurs noté, à la CGT aussi, un attrait particulier pour les positions des jeunes syndicalistes allemands.

A Force Ouvrière, les contacts avec le DGB ne sont pas chose nouvelle, puisque FO adhère à la CISL. Les contacts bilatéraux existent aussi sur divers sujets; l'une des dernières rencontres a eu lieu sur les problèmes de la sécurité sociale et particulièrement de l'assurance vieillesse.

On constate donc partout, mais peut-être davantage dans les syndicats français qui avaient été jusqu'ici les plus critiques, un désir de mieux connaître le syndicalisme étranger et en particulier le DGB. Cette orientation nouvelle semble particulièrement nette à la CFDT.

\section{COMPARAISON ET APPRÉCIATION DES PRATIQUES REVENDICATIVES}

Le terme «négociation collective» n'a pas le même sens en France et en Allemagne. Ce mot, que la pratique syndicale américaine a transformé en slogan, est trompeur parce qu'il recouvre des réalités très différentes et on peut craindre que son usage trivial dans le vocabulaire des grandes organisations internationales (OCDE, BIT, CEE...) n'entraîne plus de confusion que de clarté. La pratique allemande repose sur le principe de l'autonomie des partenaires sociaux et sur la technique des contrats à durée déterminée. Quant à l'autonomie des partenaires sociaux, il est vrai que la crise économique rend de plus en plus décisive, sur la situation sociale, l'action économique du Gouvernement et que les syndicats ne peuvent s'en désintéresser. Mais l'intervention active des pouvoirs publics dans la négociation et la conclusion des accords est vivement refusée par les employeurs comme par les syndicats en Allemagne. Au contraire, en France, depuis 10 ans, de nombreux accords généraux (pour l'ensemble de l'industrie et du commerce): sur la réduction de la durée du travail, les garanties sociales en cas de licenciement, la formation professionnelle, l'égalisation des avantages sociaux des ouvriers et des employés («mensualisation») etc... ont été conclus à l'initiative et sous la pression des pouvoirs publics (pression généralement exercée sur les employeurs). Il est important d'ailleurs de noter qu'il s'agit là d'accords de contenu (substantive issues) et non d'accords sur les procédures ou sur les institutions, domaine dans lequel, dans tous les pays, l'État joue un rôle important. 
Dans le domaine des négociations de salaires, les syndicats ouvriers manœuvrent depuis 1975 , entre les directives gouvernementales sur les hausses maxima et la fixation autoritaire du salaire minimum national. Mais la marge de manœuvre est étroite dans la mesure même où le système de conventions à durée indéterminée permet les atermoiements des employeurs, surtout en période de chômage. Finalement, tout le système repose sur la capacité syndicale d'action directe dans les entreprises. Une grande entreprise ou une industrie prospère et pourvue de commandes voudra éviter les grèves et les ralentissements de production en accordant des hausses, même si l'organisation patronale ne signe aucune convention de salaire. Au bout d'un certain temps, la généralisation des hausses dans les entreprises obligera l'organisation patronale à généraliser ces hausses en proposant aux syndicats un accord de salaires... que ceux-ci refuseront généralement de signer, et qui se transformera en recommandation unilatérale de l'organisation patronale à ses adhérents: alors les employeurs qui ont pu résister jusque là aux syndicats d'entreprise l'appliqueront.

On peut d'ailleurs se demander si cette technique ne s'explique pas uniquement par le pluralisme syndical: comme la concurrence que se font les syndicats s'exerce principalement dans les grandes entreprises, où les 3 organisations ouvrières sont généralement représentées, et comme chacune «organise» un ou plusieurs groupes particuliers de travailleurs, il s'agit pour l'un des syndicats de saisir l'occasion d'obtenir, le premier, pour ce ou ces groupes, une augmentation de salaires, éventuellement appuyée par des grèves courtes mais répétées. Cette augmentation de salaires fera rapidement tache d'huile sur tout le personnel de l'établissement. Ce système a été assez efficace malgré la crise, puisque jusqu'en 1975, les salaires réels ont augmenté d'environ 4\% par an (encore en 1975). Ce système est malheureusement assez inflationniste. Ce n'est qu'à partir de 1976 que le Gouvernement a demandé aux employeurs - et a réussi à obtenir - le blocage de la hausse du pouvoir d'achat.

Il en irait évidemment tout autrement si, comme en Allemagne, un seul syndicat négociait des conventions à durée déterminée. Les syndicats français ne nient pas d'ailleurs que le système allemand ait, lui aussi, une grande efficacité: ils sont assez impressionnés par les salaires allemands et parfaitement conscients que, sur la longue période, les résultats obtenus sont encore meilleurs que ceux du système français. Mais il est difficile de dire si cette efficacité vient plutôt de l'efficacité des syndicats ou de l'efficacité générale de l'économie allemande. 
Quel que soit le jugement sur l'efficacité, il est cependant un point sur lequel les syndicats français - spécialement la CGT, mais sans doute aussi la CFDT - pensent que leur système a un avantage sur le système allemand: le caractère non formalisé et même quelque peu anarchique de la lutte pour les salaires a une conséquence sur les relations entre les syndicats et les travailleurs. La recherche par les syndicats, de la mobilisation permanente des travailleurs - et, corrélativement, la dépendance des syndicats par rapport à la volonté de lutte des travailleurs - entretient, pensent-ils, une interaction plus forte et plus continue entre l'organisation syndicale et sa base. Il faut, pour la section syndicale d'entreprise, être constamment à l'écoute des revendications et constamment prête à mobiliser les travailleurs. Un système plus formel de négociation risque d'entraîner un certain degré de rigidité, de bureaucratie, d'éloignement entre les travailleurs et leurs syndicats. Là encore, il est facile de voir le lien étroit entre cette attitude et le pluralisme syndical, puisque le risque de manquer une occasion de mobilisation est en partie fondé sur le danger que le syndicat concurrent la saisisse.

Les syndicats français sont d'ailleurs conscients du problème que leur pose cette situation: c'est le problème du contrôle de l'action de la base, le problème d'assurer la cohérence de la politique syndicale. Mais comment y échapper lorsque, au pluralisme syndical, s'ajoute le fait que les gouvernements, depuis 20 ans, suivent une politique plutôt favorable aux employeurs qu'aux syndicats? Si un renversement de la tendance politique se produisait, il ne fait guère de doute que les syndicats tenteraient d'assurer un plus grand contrôle sur leur base et combattraient le trop grand «spontanéisme» de certains groupes de travailleurs. Alors, si - condition supplémentaire et nécessaire - la concurrence syndicale s'atténuait ou disparaissait, la situation et la politique des syndicats français deviendraient peut être plus proches de celle du DGB.

Les syndicats français - au moins certains d'entre eux - sont, de plus, très conscients du fait que leur stratégie sociale de mobilisation maximum est liée à leur relative faiblesse - faiblesse ellemême liée, encore une fois au pluralisme - En effet, un ralentissement de l'action tend toujours à avoir des effets cumulatifs: découragement des «militants», indifférence des adhérents ordinaires, contreoffensive des employeurs. En quelque sorte, il faut que la vitesse et la mobilité remplacent l'effet de masse. Une des caractéristiques du syndicalisme français depuis un demi-siècle, ce sont les alternances de forte poussée revendicative, de forte adhésion syndicale, d'une part, 
et de retombées dans la stagnation de l'action et de l'adhésion. C'est seulement lorsqu'on est fort qu'on peut se permettre d'être pondéré et de négocier des compromis durables. Ce n'est pas que les syndicats français soient opposés à la négociation; c'est que pour eux, elle est plutôt le moyen que le résultat de l'action.

Ceci permet aussi de comprendre leur attitude à l'égard de la cogestion.

\section{LE PROBLÈME DE LA PARTICIPATION À LA GESTION}

L'attitude des syndicats allemands à l'égard de la cogestion a été l'objet à la fois d'intérêt et de critique. La critique est d'ailleurs à peu près unanime. Elle va de la CGT à Force Ouvrière. Mais il faut noter que cette attitude critique des syndicats français ne leur est pas particulière; elle existe aussi chez les syndicats américains de l'AFLCIO. Et ces critiques ont deux points communs : d'abord la Mitbestimmung, ne ferait pas une place suffisante au syndicat lui-même mais fonderait trop les représentations ouvrières qu'elle accueille sur des votes directs du personnel, où la liste syndicale peut exister à côté d'autres listes non syndicales; ensuite, l'idée même de participation à la gestion rebute la plupart des syndicalistes, qui voient là un moyen, pour l'employeur, de faire prendre au syndicat des responsabilités dans des domaines où la décision économique - toujours fondée sur des contraintes (vraies ou fausses) de marché - lui échappe toujours finalement. Il y a quelque chose de juste dans ces deux critiques. Les syndicats français tendent cependant à se rendre de mieux en mieux compte que les syndicats allemands sont eux-mêmes conscients de ces problèmes, qu'ils essaient d'éviter les inconvénients de leur situation et que, de plus, cette situation doit être interprétée dans le contexte historique, politique et social de l'Allemagne d'aujourd'hui.

D'abord, spécialement à la CFDT, on se rend compte que l'attitude générale à l'égard de la participation à la gestion dans la société capitaliste ne peut se comprendre qu'en tenant compte de la force même du syndicat. De même que, dans le problème de la négociation de salaire, l'engagement contractuel est souvent un piège pour un syndicat faible, de même, dans le problème plus général de la participation aux décisions, le poids social du syndicat dans la société est un facteur essentiel. En effet, tout dépend du prix que les employeurs ou les pouvoirs publics devront payer au cas où ils ne respecteraient pas leurs propres engagements. Dans le cas d'un syndicalisme faible, ce prix sera lui même très faible. Quand le personnel est syndiqué à 
moins de $50 \%$ dans une entreprise ou dans une industrie, il suffit que la situation économique devienne effectivement difficile pour que l'influence décisive sur le personnel passe aux employeurs: d'une part, il est peu probable que les solutions syndicales soient prises en compte dans l'entreprise, l'industrie, ou la nation. D'autre part, la démonstration des «contraintes économiques» sera facile et permettra aux employeurs de remettre en cause leurs engagements. Le pluralisme syndical, là encore, ne fait qu'aggraver ce danger. Au contraire, on se rend compte, en France, que dans des pays comme l'Angleterre, l'Allemagne, mais aussi l'Italie, le poids social et politique des chefs syndicaux est considérable: Vetter, Jones et Lama sont, à bien des égards, les « $\mathrm{n}^{\circ} 2$ » de l'économie dans leurs pays en ce sens qu'une décision anti-syndicale ou anti-ouvrière peut provoquer une mobilisation immédiate, certaine et durable, de tous les travailleurs. C'est peut-être plus vrai encore en Allemagne et en Italie qu'en Angleterre mais dans les trois cas, c'est plus vrai qu'en France, où les «grandes journées nationales" sont devenues un rite, voire une fête qui n'impressionne guère les pouvoirs économiques et politiques de droite. C'est dans la mesure où les pouvoirs publics et les employeurs allemands ont réellement besoin de l'accord des syndicats qu'ils doivent prendre au sérieux leurs engagements à leur égard, de crainte de perdre leur soutien nécessaire. En France, la plupart du temps, tant à cause du pluralisme que de la faiblesse globale du syndicalisme, employeurs et Gouvernement peuvent généralement se passer de l'appui des syndicats. C'est pourquoi ceux-ci peuvent facilement afficher une attitude de refus énergique de toute participation. D'ailleurs, un trait du système français de relations industrielles est que le refus de cogestion est aussi vivement exprimé par les patrons que par les syndicats. Il faut cependant noter que le Gouvernement prend parfois une attitude différente et exige tacitement - du patronat qu'il obtienne dans certains cas l'accord de tous les syndicats. Un des exemples est celui de la convention sociale de la sidérurgie de l'Est, en 1967, par laquelle les pouvoirs publics, qui accordaient aux employeurs de la sidérurgie des crédits très importants pour la restructuration de l'industrie, demandèrent, que des garanties d'emploi précises (reclassement, formation, retraites anticipées) soient accordées aux travailleurs dans un accord (sorte de Social plan) et que ces garanties soient inscrites dans une convention discutée et signée par tous les syndicats. Les discussions furent longues, et même une grève eut lieu. Mais, comme chaque partenaire avait intérêt à ce que la convention soit conclue, elle le fut finalement, et par tous les syndicats. On a là un exemple qui montre que, même dans le cas des syndicats français, lorsque la force leur est donnée - lorsque 
leur accord est nécessaire - ils ne refusent pas absolument une certaine participation aux décisions.

C'est encore plus net dans le cas des entreprises nationalisées et du pouvoir des comités d'entreprise. Les entreprises nationalisées créées en 1945 étaient administrées par un Conseil tripartite composé de représentants des syndicats, de représentants des consommateurs, de représentants de l'État. En fait, très rapidement, l'État, (en nommant lui-même une partie des représentants des consommateurs) s'est assuré une prérogative absolue dans les Conseils et le rôle des syndicats est devenu négligeable. Or, un des projets actuels les plus importants des syndicats est de renforcer leur rôle dans ces entreprises selon des formules qui impliquent une participation à leur gestion. La CGT, dès 1973, avait établi un texte sur «la gestion démocratique des entreprises» où on lisait: «le principe de la composition tripartite des conseils (élus des travailleurs, représentants de certaines catégories d'usagers, représentants du pouvoir dérnocratique ${ }^{4}$ ) ne signifie pas la division par tiers. La représentation des travailleurs doit être importante. La CGT estime que la représentation des usagers au titre de la population (organisations démocratiques et collectivités élues) jointe à la représentation élue des travailleurs, devrait former la majorité ${ }^{5}$. On voit donc que la CGT est loin de refuser la participation à la gestion, pour des entreprises nationalisées et dans le cas d'un gouvernement de gauche, même dans un système capitaliste. Dans ce cas très favorable, les représentants des travailleurs accepteraient d'avoir la majorité dans les conseils. Même le simple retour à la situation de 1945 (véritable représentation d'un tiers pour les seuls travailleurs, à laquelle s'ajouteraient certains éléments élus au titre de la représentation des consommateurs) serait encore considéré comme un progrès. C'est une revendication constante, depuis que l'État a tourné la règle à son profit en 1953. Enfin, si de tels conseils d'administration à majorité ouvrière voyaient le jour, «toutes les décisions importantes» (en matière économique, technique, financière, commerciale, dans le cadre des directives données par le Plan Économique) «seront prises par les Conseils d'Administration et sans intervention autoritaire des ministères de tutelle ${ }^{6}$. Il en va de même naturellement dans le domaine social: pour les rémunérations, les conditions de travail, etc... sur la base de négociations syndicales. Même si ce progranme peut

4 Les auteurs veulent dire ici: «l'Etat» et pensent au cas où des élections donneraient le pouvoir à la gauche dans une perspective approuvée par la CG'T (F.S.)

5 Le Peuple, 15-31 mai 1977, p. 20.

6 Ibid., p. 13. 
être considéré comme un objectif maximum, il est cependant certain que l'intérêt considérable porté par le CGT et la CFDT pour de nouvelles nationalisations est lié étroitement au pouvoir, même non majoritaire, qu'ils détiendraient dans les conseils d'administration et, grâce à cette influence, à la croissance et à la sécurité qu'y gagneraient les syndicats. Dans les industries nationalisées en 1945, le taux d'adhésion syndicale est nettement plus élevé que dans les entreprises du secteur privé, même dans les plus grandes et les plus syndiquées. Enfin, tous les syndicats sont d'accord pour l'augmentation des pouvoirs des comités d'entreprise dans toutes les entreprises, nationalisées ou non. Il s'agit, comme le précise un texte de la CGT de 1977, de donner aux comités d'entreprise «un droit de recours suspensif pour tous les projets d'une importance telle qu'ils affectent la structure de l'entreprise et sa marche globale pour une certaine durée». Sans doute, pour la CGT, ces projets sont liés à un changement de gouvernement, mais non à un changement complet du système économique.

La CFDT est sans doute plus prudente et plus réticente à proposer des mesures de participation à la gestion, même dans des industries nationalisées. Elle insiste davantage que la CGT sur une forte décentralisation de l'exercice du pouvoir de contrôle des travailleurs («autogestion»). Le syndicat CFDT de la métallurgie va même jusqu'à écrire: «(il faut) éviter toute confusion entre les différents pouvoirs dans l'entreprise nationalisée; le syndicat ne doit pas avoir de responsabilité de gestion ${ }^{7}{ }^{7} .$. Mais aussi: «au sein du conseil d'établissement, les travailleurs doivent être assurés d'exercer progressivement l'ensemble du pouvoir d'orientation et de décision, spécialement pour les conditions de travail et l'organisation du travail, par des structures décentralisées disposant d'une autonomie et de pouvoirs négociés avec le conseil d'administration.

Force Ouvrière est autant, voire plus réticente que la CFDT dans le domaine de la participation à la gestion et insiste sur la prééminence de la seule négociation par le syndicat lui-même et sur le «contrôle ouvrier». Mais il s'agit là aussi d'une position qui s'explique par l'environnement social et syndical.

Paradoxalement, en effet, le syndicat qui insiste le plus sur les pouvoirs de gestion des travailleurs, c'est la CGT, puis à un moindre degré la CFDT, enfin au degré zéro, F.O. Ces positions sont étroitement liées à l'influence relative de ces syndicats. Partout, ou presque

7 Bulletin du Militant, FGM, n 73 , octobre 1977, p. 36. 
partout, la CGT a la majorité. Le pouvoir de gestion des travailleurs, serait donc, dans une large mesure, son propre pouvoir. C'est pourquoi les syndicats minoritaires sont plus prudents. On a l'impression que, souvent, leur opposition à la cogestion allemande, c'est l'opposition au risque que présenterait, pour eux, l'importation pure et simple du système allemand dans la situation française.

\section{How French Labour Unions View the Policies of the D.G.B.}

This paper aims at exposing and clarifying the differences between various systems of industrial relations in Western Europe, mainly between the French system and the German one.

Many aspects of these two systems are common, even though the experience is pursued throughout various means and methods: defence of workers, quest for control on management powers to decide, influence to be exterted on the legislative and economic regime.

A closer approach is pursued between unions of France and Germany, and the latter is favoured by the actual economic crisis which bears on the economics and on the workers of both countries, and by the obligation of the unions to negotiate with multinational corporations. This effort is visible in France where the three main unions, CGT, CFDT and FO, in spite of certain differences between them, favour a trend towards widening European labour cooperation.

This widening appears on two particular grounds: the appreciation of claim applications of both movements and the question of participation in management.

On the first subject, it is obvious that the term "Collective bargaining" has a different meaning in France and in Germany.

Even if the actual economic crisis draws together both movements, the intervention from the administration into the bargaining process and the ratification of agreement are turned down by the unions and the employers in Germany. Whereas, in France, for the last ten years, many general settlements regarding reduced workweek, social securities in any case of layoff, vocational training, levelling of fringe benefits between white-collars and workmen have been endorsed.

Regarding wage-setting, things are different in both movements. Owing undoubtedly to the inter-union rivalry, French unions consider their system more beneficial than the German system, since their somewhat anarchical fight for wage increases enables them to consider at any time the workers' claims; whereas the German regime, more formal, implies a certain severity, some red-tape and an greater distance from a unions. In France, the plural unionism favours such procedure, but it does not mean that French unions 
are not aware of that question of control of rank and file action, and there is no need for them to look after a more consistent union policy.

Conflict of opinions of both regimes is reflected upon the problem of participation in management.

The attitude of German unions towards co-determination raises at the same time interest and critic from French unionists. It is taken to task for not making enough room to unions, because participation in management is based upon direct votes from the staff rather than from rank and file. They also consider that this form of participation is naturally discouraging union members who wee a means for the employers to get the unions to accept responsibilities in spheres where decisions based on restrained deals slop out from them. It is said that where the union is weak, it is enough for the economic situation to become tight to see decisive influence pass out of their hands to the employers'.

Moreover, it is clear that French unions with their acquired power do not reject a certain participation in decision making. For instance, in the nationalized firms, to oppose the State prerogatives, unions try to strenghten their role in them. CGT particularly does not give up searching in those fields to a form of representation in which it would succeed in reaching the majority with the help of consumers's representatives. CFDT is far more unwilling; it rather aims at decentralizing the use of control-power, in other words, self-management. FO, closer to the American concept of collective bargaining, gives preeminence to the latter and dismisses the participation in management to workers.

\section{LES RELATIONS DU TRAVAIL AU QUÉBEC}

\section{La dynamique du système}

Introduction, Jean BERNIER, Rodrigue BLOUIN, Gilles LAFLAMME, Alain LAROCQUE - Où s'en va notre système de relations du travail?, Jean BERNIER - L'injonction en relations du travail: recours inapproprié ou abusif?, Henri GRONDIN - Commentaires, Jean BEAUVAIS, Philip CUTLER - Méditations politiques, commissions parlementaires et lois spéciales: nouveaux modes de gestion des conflits?, Fernand MORIN - Interventions accrues du judiciaire et du politique: leur signification pour les partenaires sociaux, Marcel PEPIN, Ghislain DUFOUR, Jean BOIVIN - Y a-t-il encore place dans notre système de relations du travail pour l'arbitrage des différends?, Rodrigue BLOUIN - La détermination des services essentiels: un préalable nécessaire à l'exercice du droit de grève?, René LAPERRIÈRE - Commentaires, Léo ROBACK, Douglas MCDONALD - Le fonctionnement de notre système de relations du travail peutil encore reposer sur la volonté des parties?, Claude RYAN - Commentaires, Paul-Gaston TREMBLAY, Fernand D'AOUST - La paix industrielle: une utopie?, Léon DION.

1 volume, 229 pages - Prix : $\$ 9.00$

LES PRESSES DE L'UNIVERSITÉ LAVAL 